\title{
Co-WC Heterojunction Nanoparticle Composites with Graphene Embedded in Porous Carbon Nanospheres as Electrocatalyst for Hydrogen Evolution Reaction
}

Yi-Yi Liu, Zhifeng Ye, Zhaogen Zhu, Zhaotong Ni, Qianqun Xu, Dingsheng Yuan*

School of Chemistry and Materials Science, Jinan University, Guangzhou 510632, People's Republic of China, E-mail: tydsh@jnu.edu.cn 


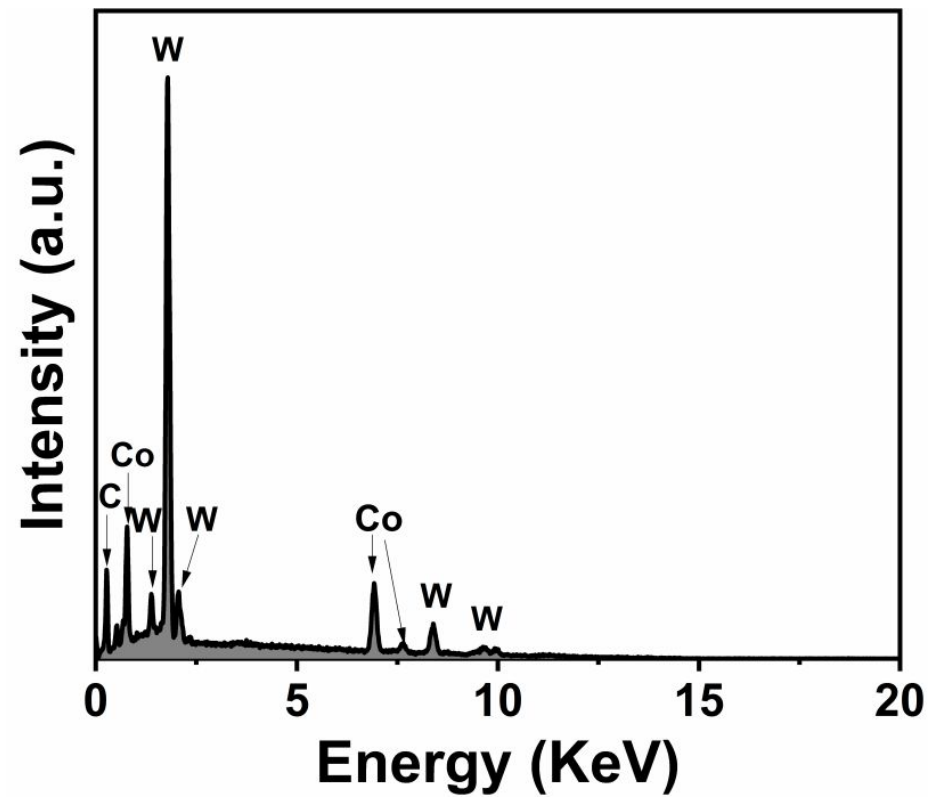

Fig. S1 The EDX spectrum for Co-WC@G/PCSs. 


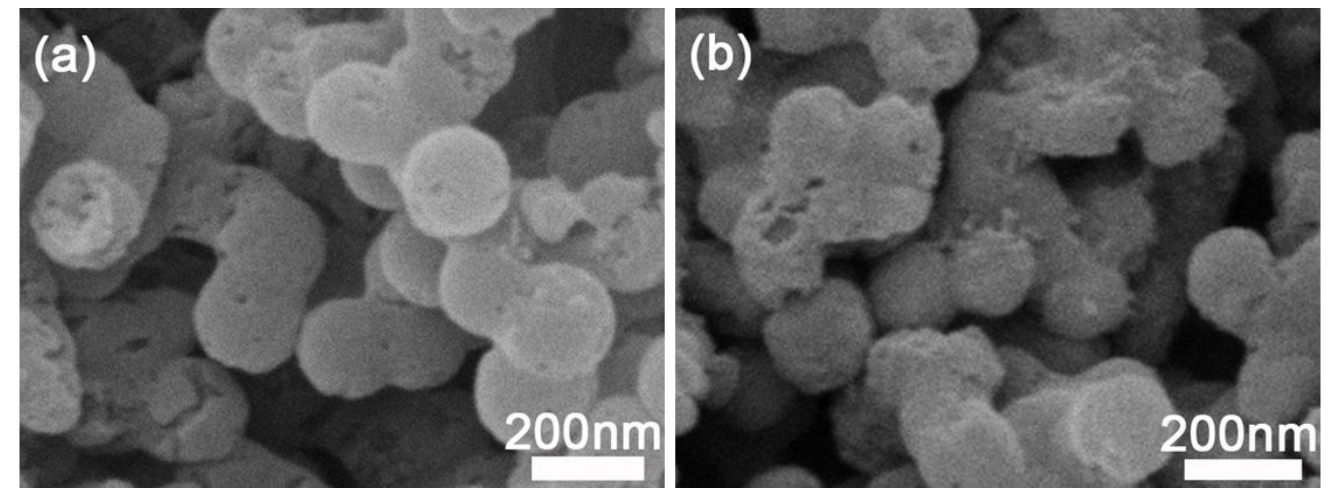

Fig. S2 SEM images of WC/PCSs (a) and Co@C/PCSs (b). 


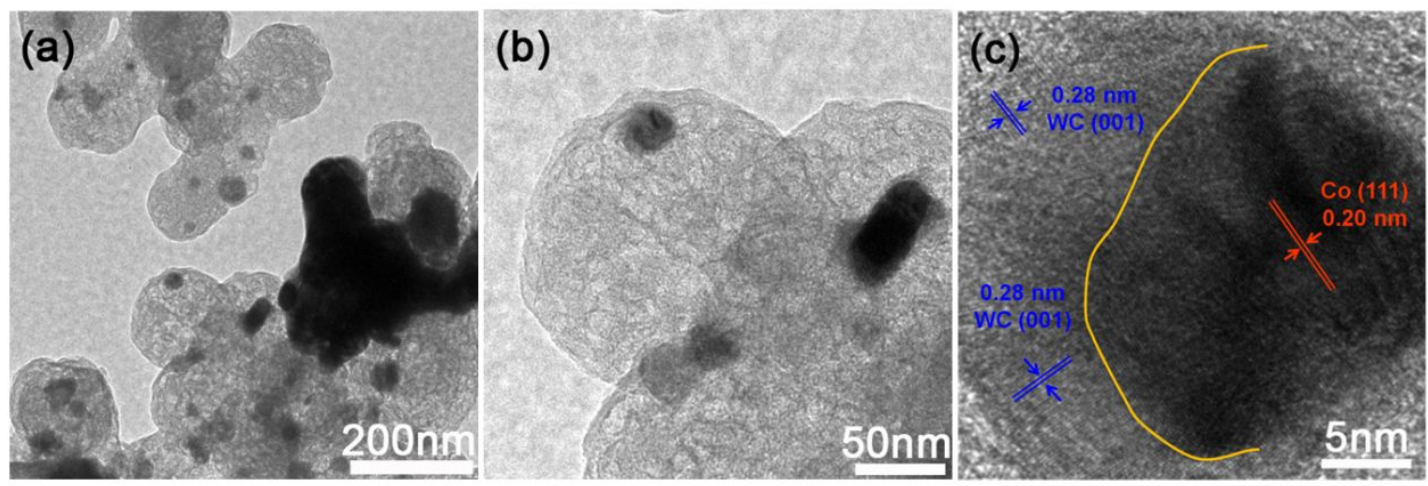

Fig. S3 (a, b) TEM and (c) HRTEM images of Co-WC@G/PCSs. 

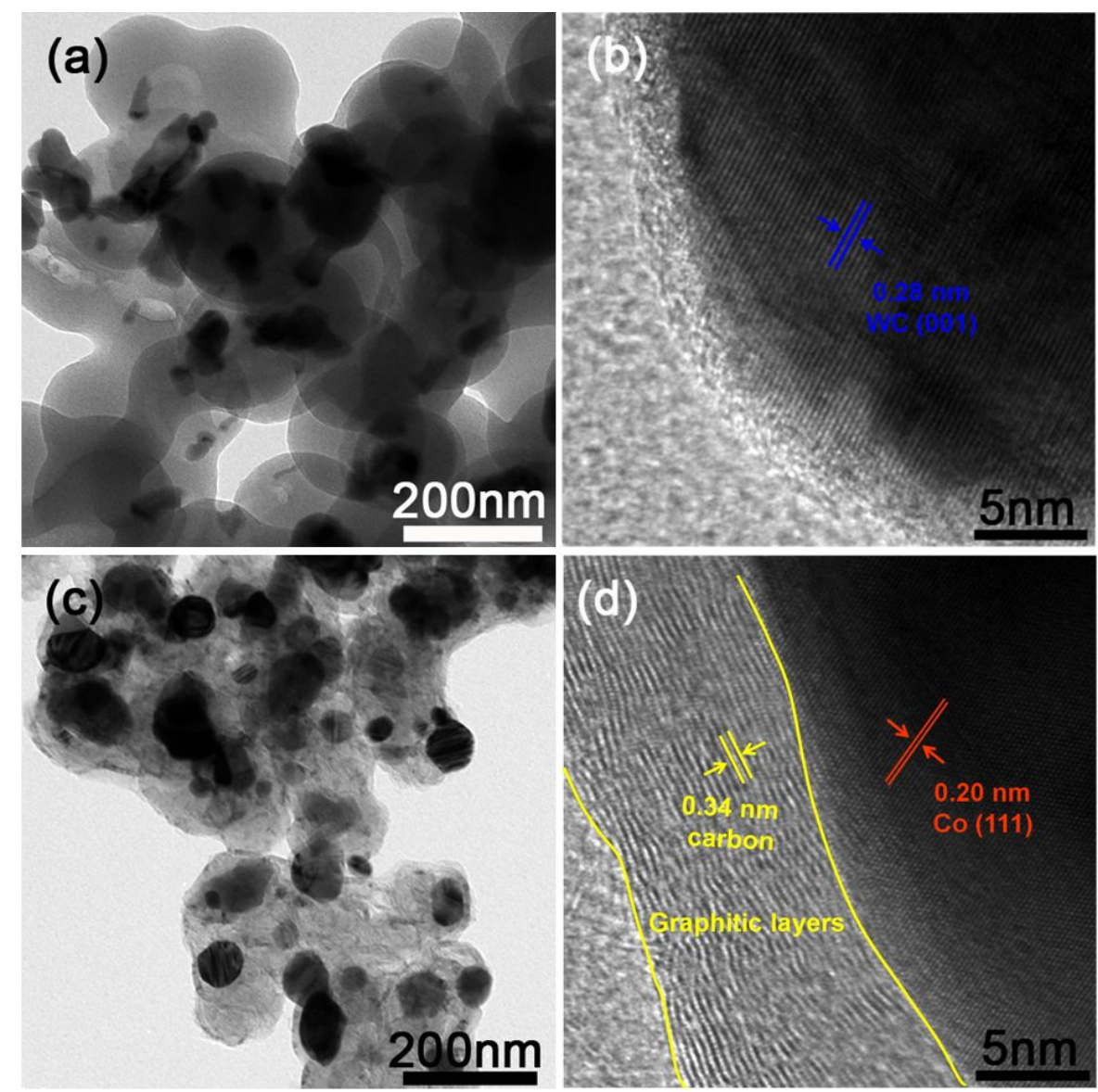

Fig S4 (a) TEM and (b) HRTEM images of WC/PCSs, (c) TEM and (d) HRTEM images of Co@C/PCSs. 


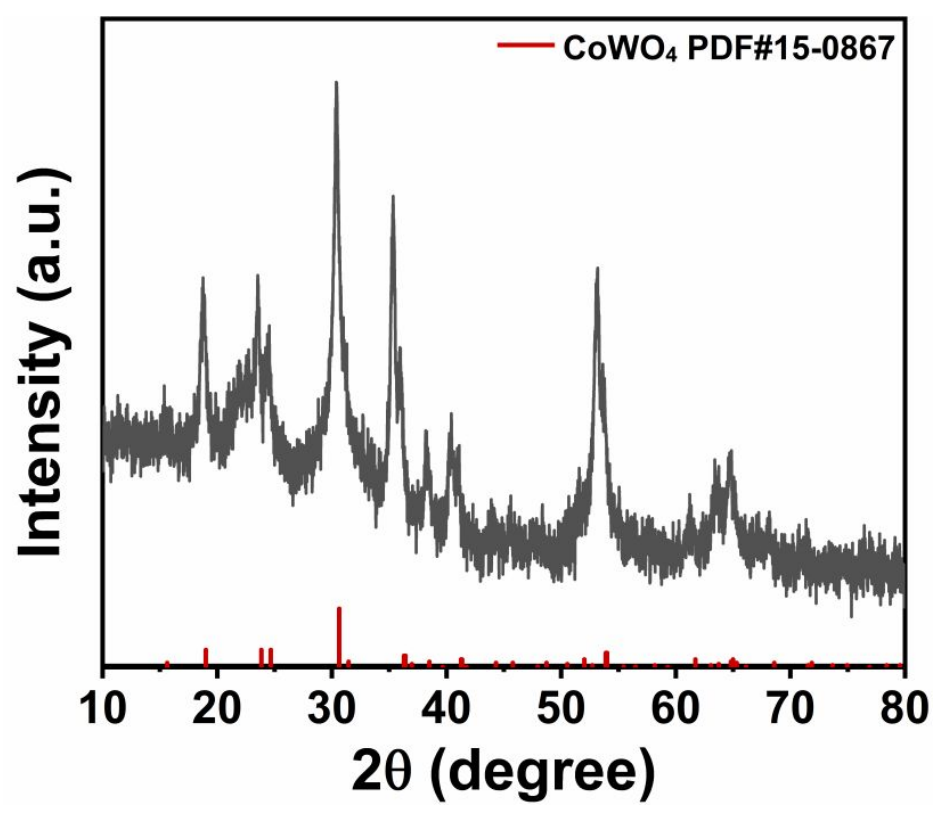

Fig. S5 XRD pattern of $\mathrm{CoWO}_{4} / \mathrm{PCSs}$. 

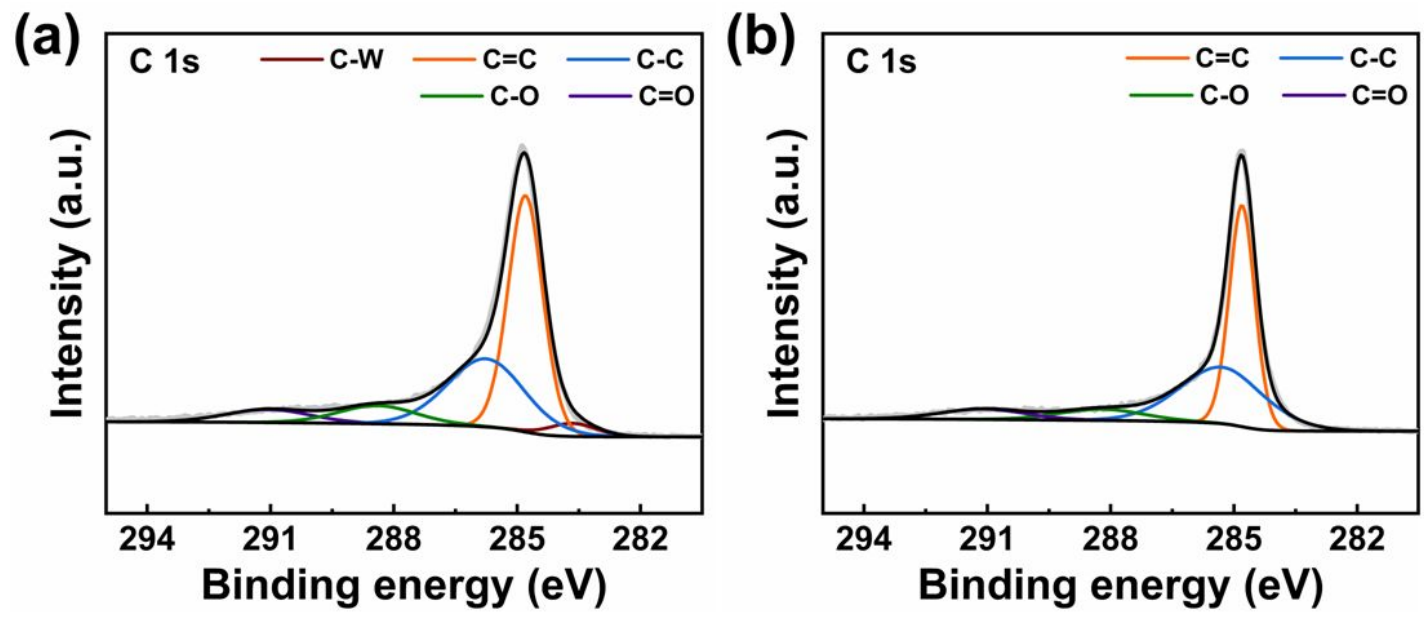

Fig. S6 The high-resolution C 1s spectra of WC/PCSs (a) and Co@C/PCSs (b). 

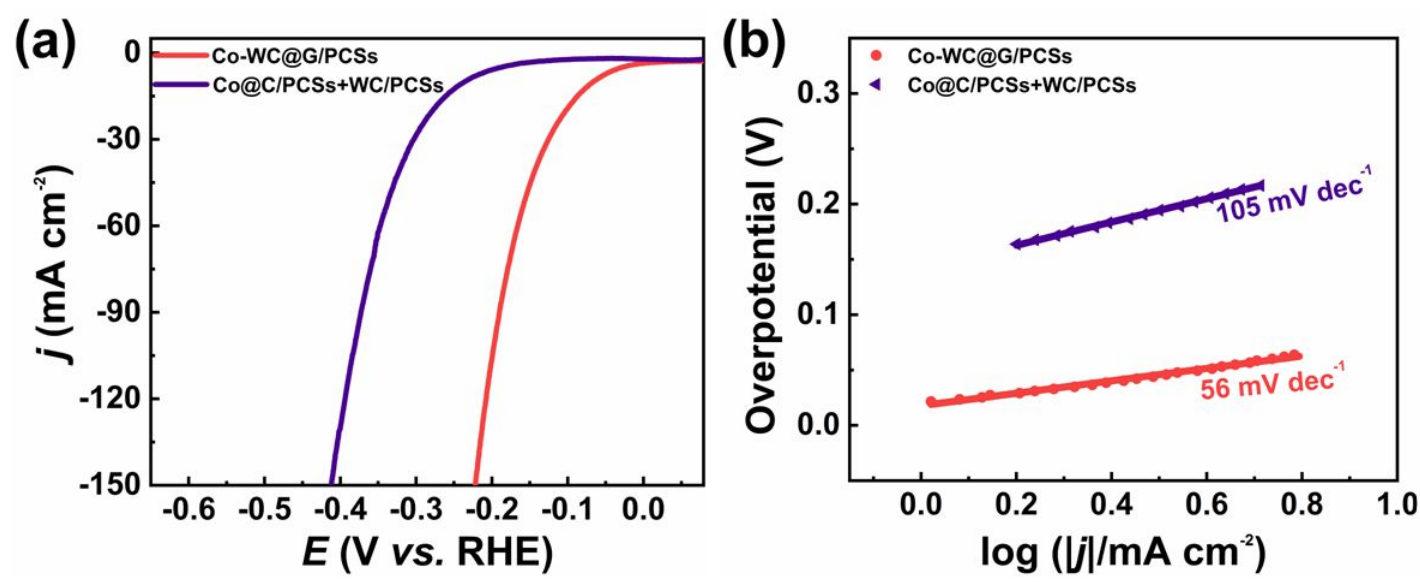

Fig. S7 (a) LSV and (b) Tafel plots of Co-WC@G/PCSs and Co@C/PCSs+WC/PCSs. 

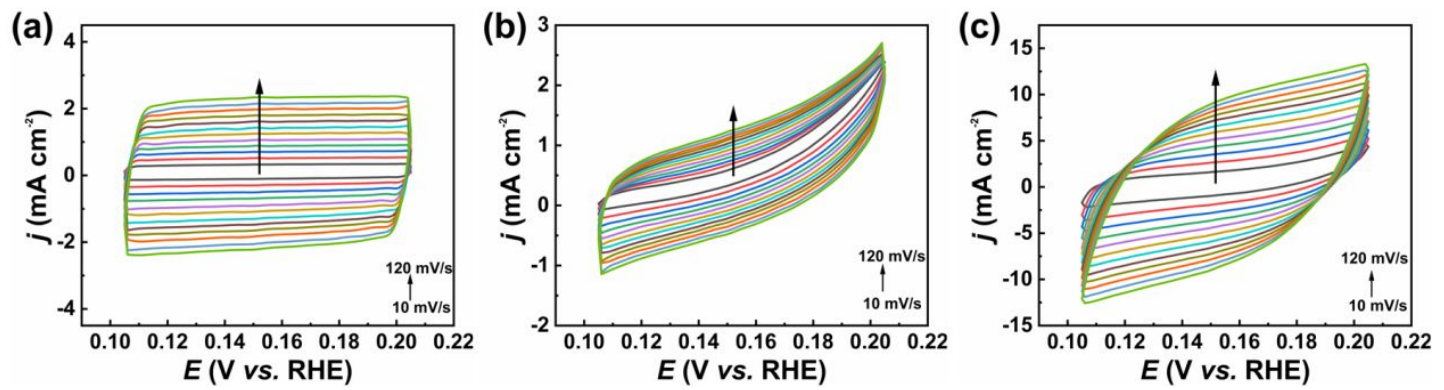

Fig. S8 CV curves of WC/PCSs (a), Co@C/PCSs (b) and Co-WC@G/PCSs (c) with different scan rates from 10 to $120 \mathrm{mV} \mathrm{s}^{-1}$. 


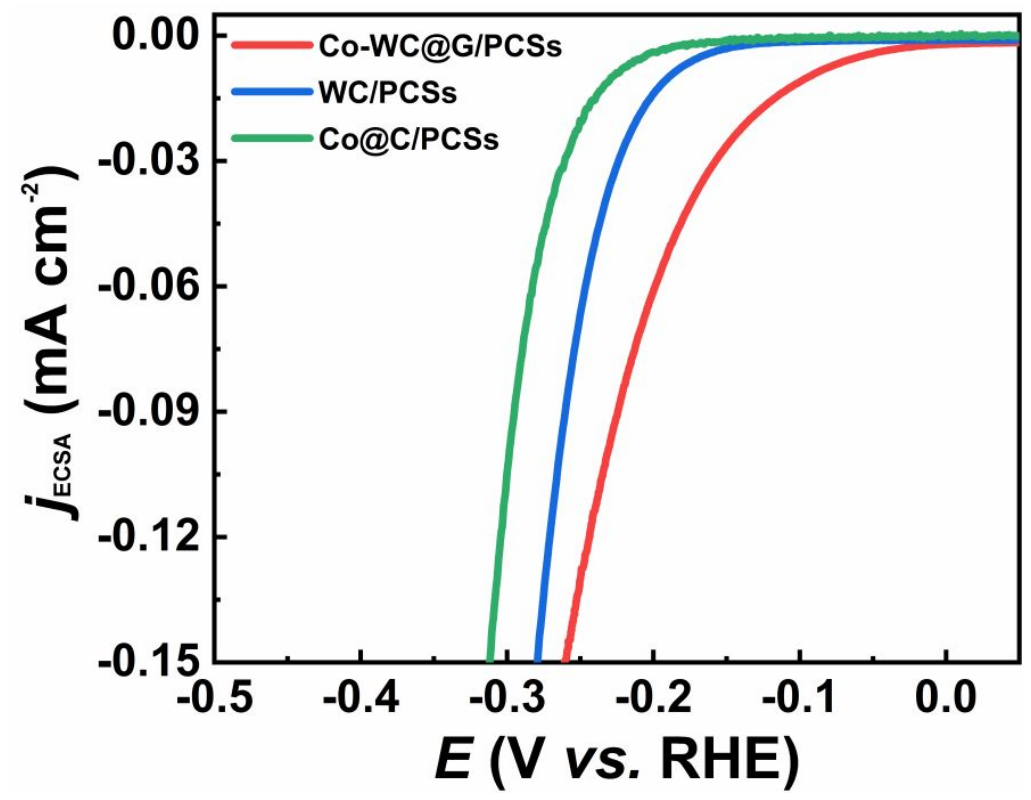

Fig. S9 LSV curves normalized by ECSA of Co@C/PCSs, WC/PCSs, and Co-WC@G/PCSs in 1.0 M KOH. 

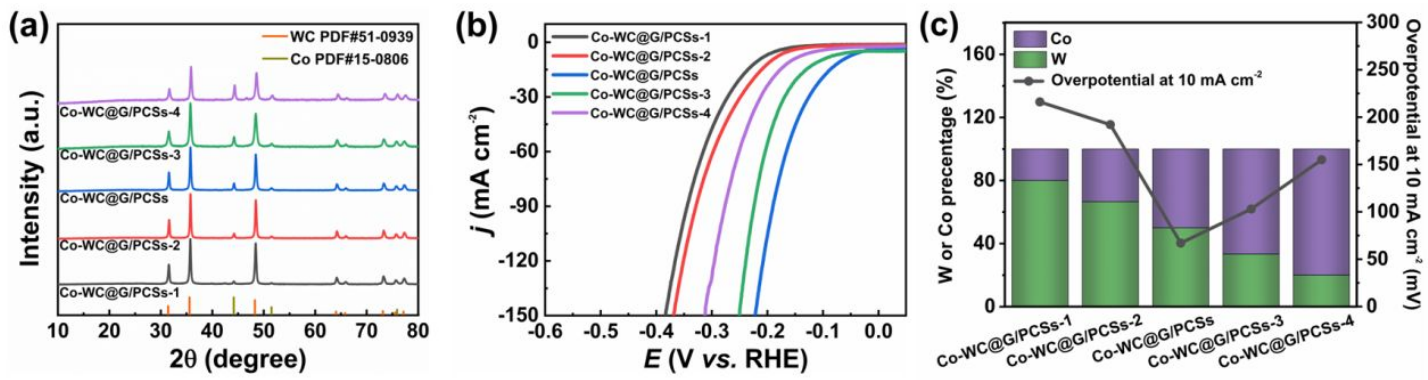

Fig. S10 (a) XRD, (b) LSV, and (c) The Co and W percentage (left axis) and overpotentials at $10 \mathrm{~mA} \mathrm{~cm}{ }^{-2}$ (right axis) curves of Co-WC@G/PCSs-1, Co-WC@G/PCSs-2,Co-WC@G/PCSs,Co-WC@G/PCSs-3,Co-WC@G/PCSs-4. 

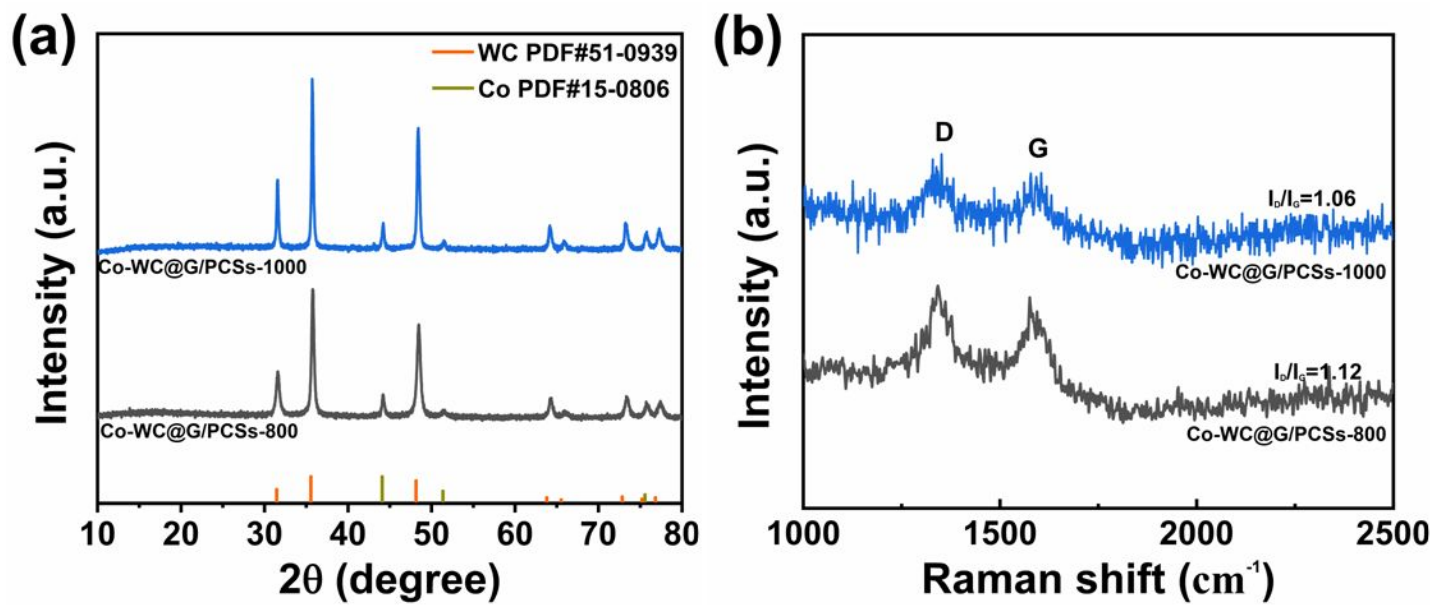

Fig. S11 (a) XRD patterns and (b) Raman spectra of Co-WC@G/PCSs-800 and Co-WC@G/PCSs-1000. 

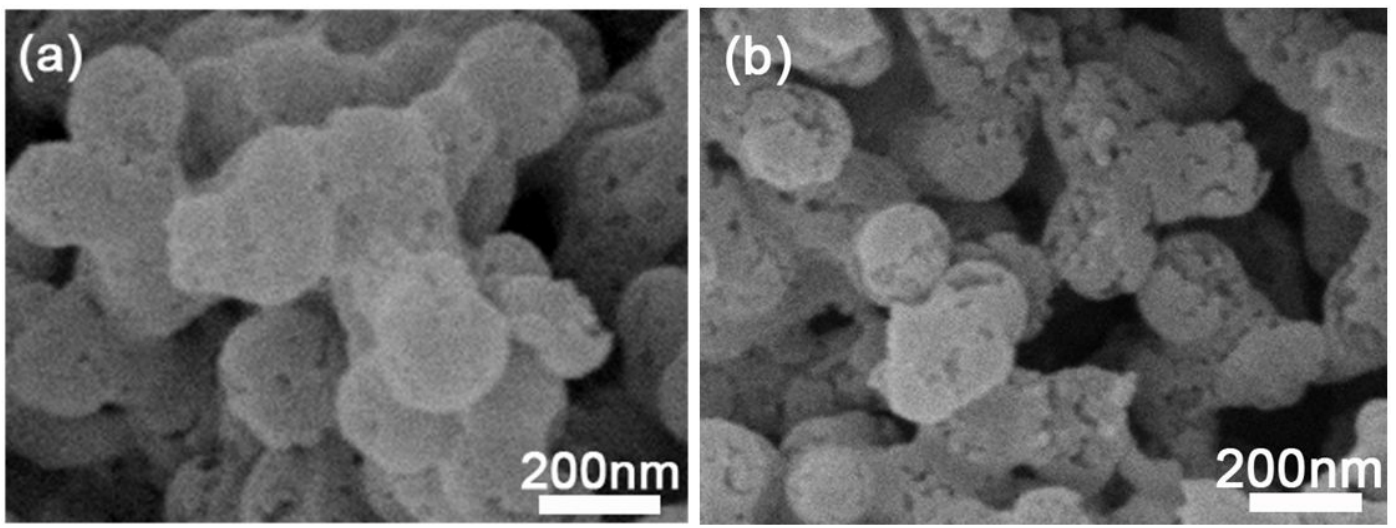

Fig. S12 SEM images of Co-WC@G/PCSs-800 (a) and Co-WC@G/PCSs-1000 (b). 

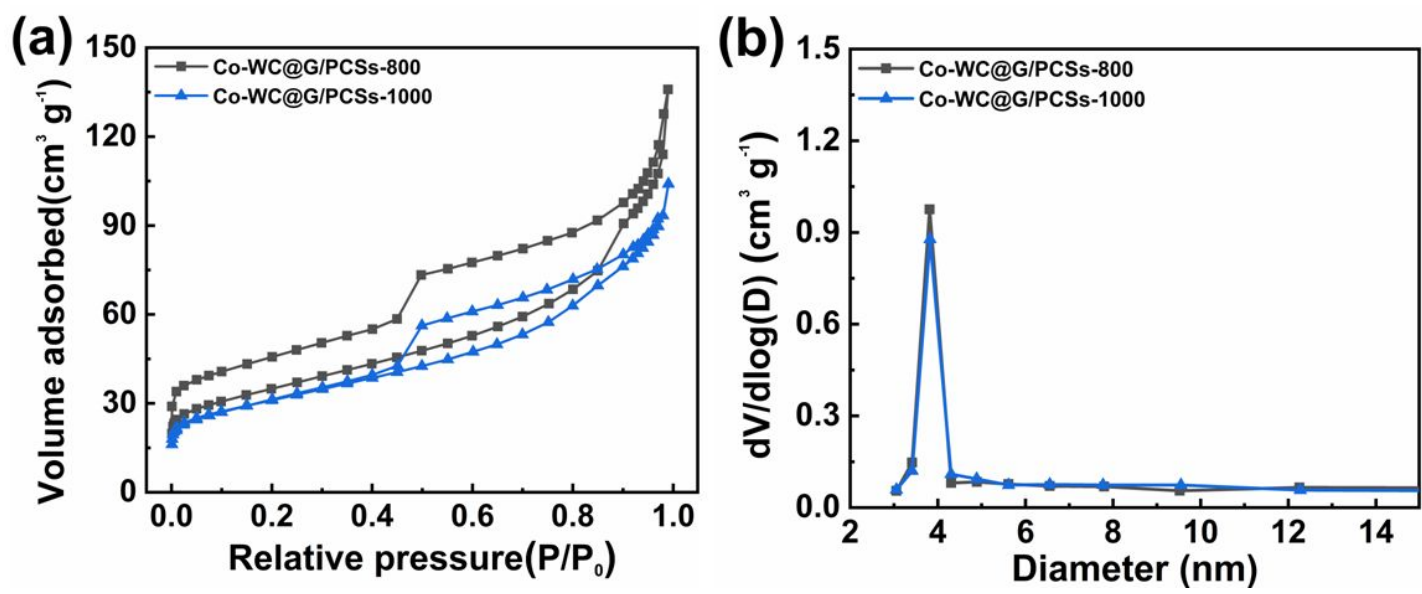

Fig. S13 (a) Nitrogen adsorption-desorption isotherms and (b) Pore size distribution curves of Co-WC@G/PCSs-800 and Co-WC@G/PCSs-1000. 

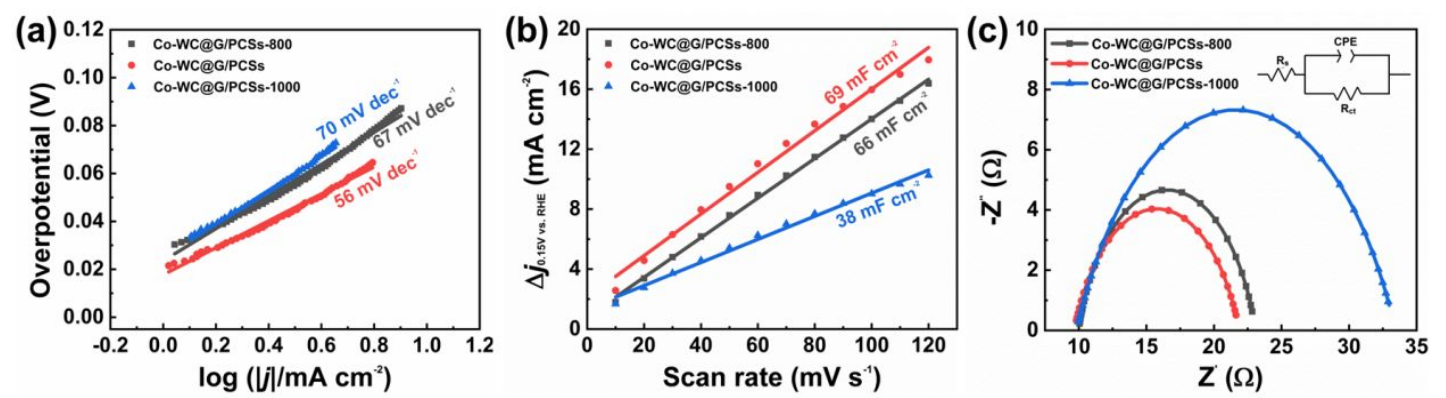

Fig. S14 (a) Tafel slopes; (b) The capacitive current at $0.15 \mathrm{~V}$ (vs. RHE) as a function of scan rate and (c) EIS curves of Co-WC@G/PCSs-800, Co-WC@G/PCSs and Co-WC@G/PCSs-1000. 

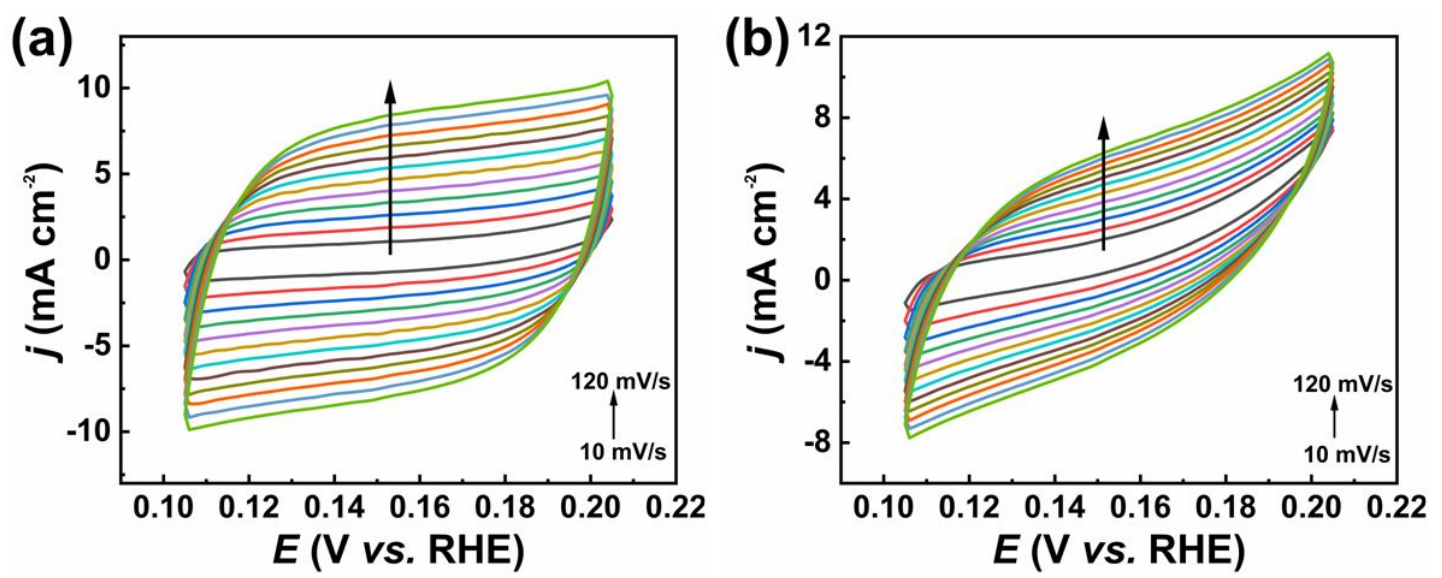

Fig. S15 CV curves of Co-WC@G/PCSs-800 (a) and Co-WC@G/PCSs-1000 (b) with different scan rates from 10 to $120 \mathrm{mV} \mathrm{s}^{-1}$. 

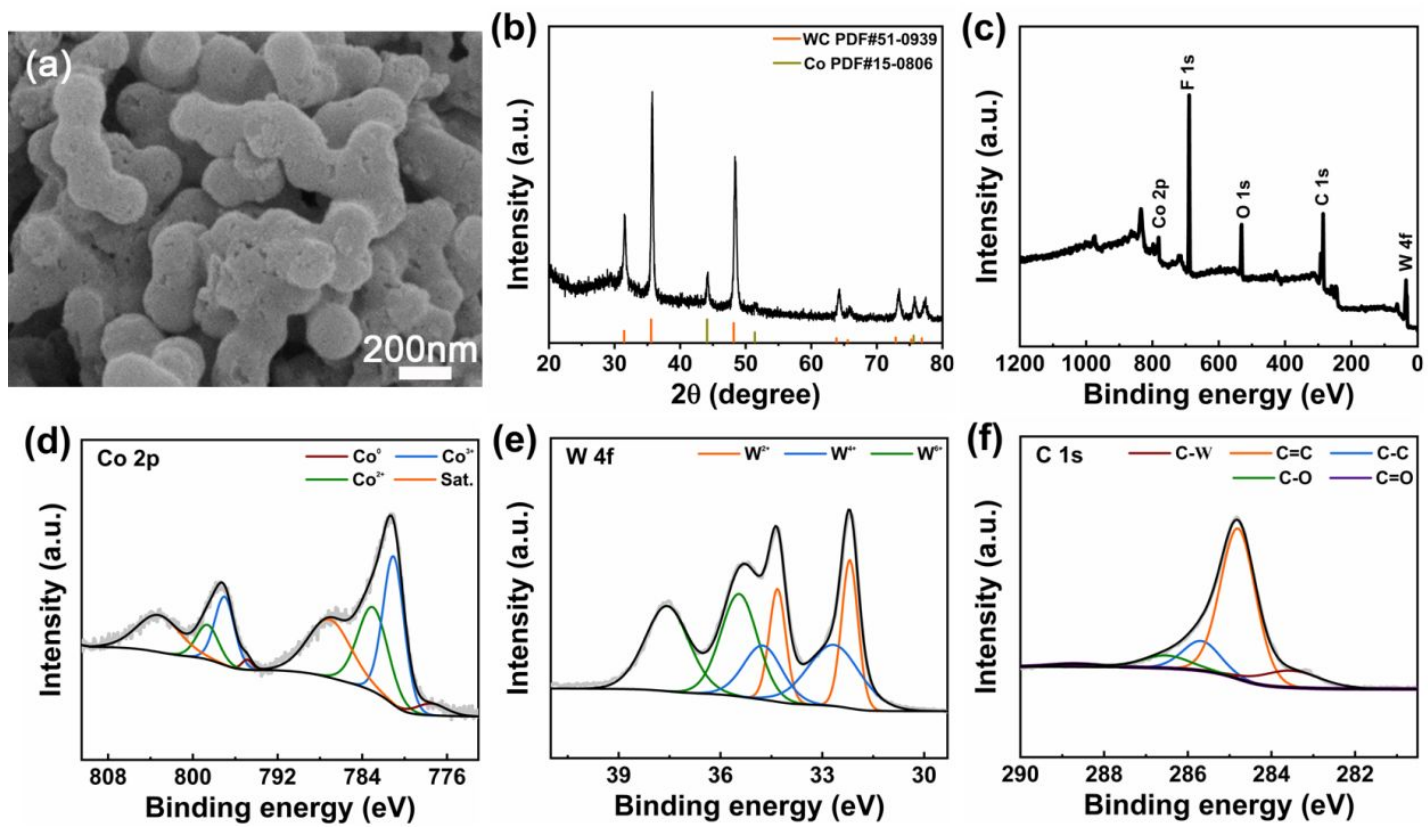

Fig. S16 (a) SEM image; (b) XRD pattern; (c) XPS survey spectrum of Co-WC@G/PCSs after HER test; (d-f) The high-resolution spectra of Co-WC@G/PCSs after HER test: Co 2p (d); W 4f (e) and C1s (f). 

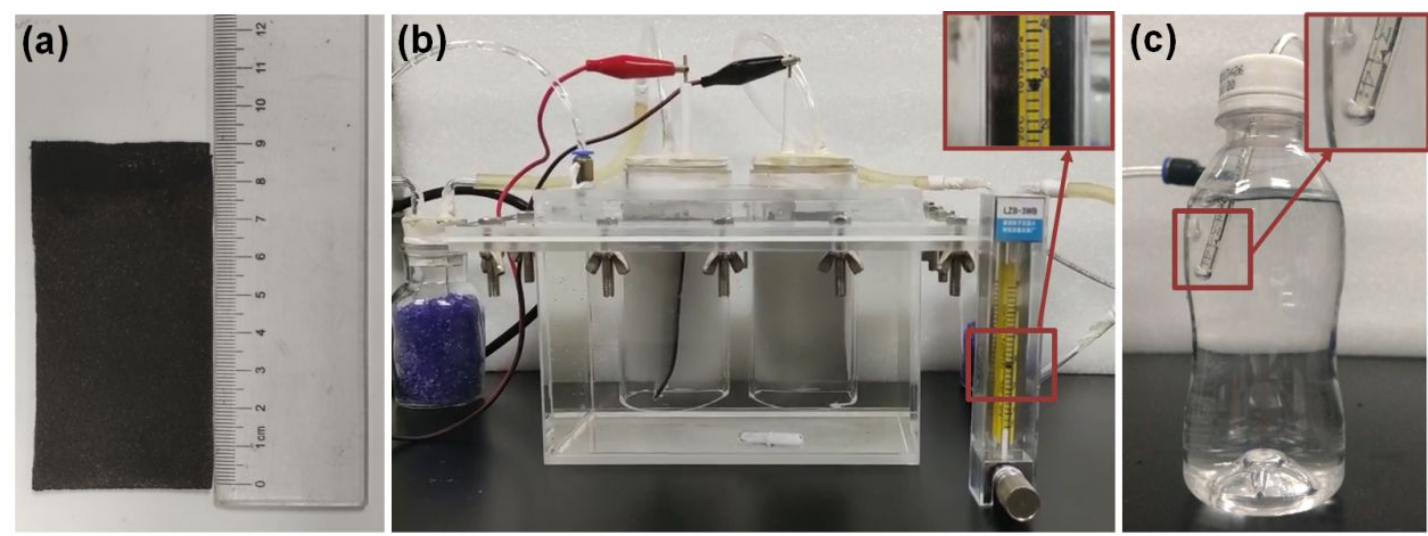

Fig. S17 (a) Photograph of Co-WC@G/PCSs-coated Ni foam electrode; (b) The device for $\mathrm{H}_{2}$ collection; (c) The hydrogen dissolved into water for rich hydrogen water. 
Table S1 HER performances of as-synthesized electrocatalysts in $1.0 \mathrm{M} \mathrm{KOH}$.

\begin{tabular}{ccccc}
\hline & $\eta_{10}$ & Tafel slope & $j_{0}$ & $R_{\mathrm{ct}}$ \\
& $(\mathrm{mV})$ & $\left(\mathrm{mV} \mathrm{dec}^{-1}\right)$ & $\left(\mathrm{mA} \mathrm{cm}^{-2}\right)$ & $(\Omega)$ \\
\hline 20wt.\% Pt/C & 36 & 35 & 0.74 & $/$ \\
Co-WC@G/PCSs & 67 & 56 & 0.48 & 12 \\
WC/PCSs & 215 & 89 & $1.9 \times 10^{-2}$ & 196 \\
Co@C/PCSs & 285 & 106 & $1.7 \times 10^{-2}$ & 277 \\
PCSs & 492 & 141 & $3.0 \times 10^{-3}$ & $/$ \\
\hline
\end{tabular}


Table S2 Comparison of electrochemical performance of Co-WC@G/PCSs and other recently reported electrocatalysts for HER in $1.0 \mathrm{M} \mathrm{KOH}$.

\begin{tabular}{|c|c|c|c|}
\hline & $\begin{array}{c}\eta_{10} \\
(\mathrm{mV})\end{array}$ & $\begin{array}{l}\text { Tafel slope } \\
\left(\mathrm{mV} \mathrm{dec}^{-1}\right)\end{array}$ & References \\
\hline Co-WC@G/PCSs & 67 & 56 & This work \\
\hline $\mathrm{i}-\mathrm{WC}-\mathrm{G}$ heterostructure & 225 & 108 & (1) \\
\hline $\mathrm{Ni}-\mathrm{WN} / \mathrm{CC}$ & 47 & 71 & (2) \\
\hline C-CoWC & 73 & 25 & (3) \\
\hline $\mathrm{Fe}_{3} \mathrm{~W}_{3} \mathrm{C} \mathrm{NRs} / \mathrm{RGO}$ & 77 & 80 & (4) \\
\hline $\mathrm{CoW} / \mathrm{CN}$ & 98 & 125 & $(5)$ \\
\hline $\mathrm{Co}_{3} \mathrm{~W}_{3} \mathrm{C} \mathrm{NSs} / \mathrm{G}$ & 84 & 84 & (6) \\
\hline $\mathrm{W}-\mathrm{W}_{2} \mathrm{C} / \mathrm{CNT}$ & 147 & 51 & (7) \\
\hline Ni/WC@NC & 77 & 68 & (8) \\
\hline $\mathrm{Co}-\mathrm{Mo}_{2} \mathrm{C}$ & 118 & 44 & (9) \\
\hline Co-WN/CC & 151 & 82 & $(10)$ \\
\hline $\mathrm{Mo}_{2} \mathrm{C} / \mathrm{C}$ & 125 & 72 & (11) \\
\hline $\mathrm{WCN}\left(\mathrm{W}_{2} \mathrm{C}-\mathrm{WN}\right)$ & 138 & l & (12) \\
\hline Co@N-CNTs@rGO & 108 & 55 & (13) \\
\hline $\mathrm{d}-\mathrm{WN} / \mathrm{Co}_{2.45}$ & 43 & 98 & (14) \\
\hline
\end{tabular}


Table S3 HER performances of Co-WC@G/PCSs with different thermal treatment temperature $\left(800^{\circ} \mathrm{C}, 900^{\circ} \mathrm{C}\right.$, and $\left.1000^{\circ} \mathrm{C}\right)$ in $1.0 \mathrm{M} \mathrm{KOH}$.

\begin{tabular}{ccccc}
\hline & $\eta_{10}$ & Tafel slope & $j_{0}$ & $R_{\mathrm{ct}}$ \\
& $(\mathrm{mV})$ & $\left(\mathrm{mV} \mathrm{dec}^{-1}\right)$ & $\left(\mathrm{mA} \mathrm{cm}^{-2}\right)$ & $(\Omega)$ \\
\hline Co-WC@G/PCSs-800 & 85 & 67 & 0.45 & 14 \\
Co-WC@G/PCSs & 67 & 56 & 0.48 & 12 \\
Co-WC@G/PCSs-1000 & 95 & 70 & 0.44 & 23 \\
\hline
\end{tabular}




\section{References}

(1) Zeng, M.; Chen, Y.; Li, J.; Xue, H.; Mendes, R. G.; Liu, J.; Zhang, T.; Rümmeli, M. H.; Fu, L. 2D WC single crystal embedded in graphene for enhancing hydrogen evolution reaction. Nano Energy 2017, 33, 356-362.

(2) Xing, Z.; Wang, D.; Li, Q.; Asiri, A. M.; Sun, X. Self-standing Ni-WN heterostructure nanowires array: A highly efficient catalytic cathode for hydrogen evolution reaction in alkaline solution. Electrochim. Acta 2016, 210, 729-733.

(3) Liu, Y.; Li, G.-D.; Yuan, L.; Ge, L.; Ding, H.; Wang, D.; Zou, X. Carbon-protected bimetallic carbide nanoparticles for a highly efficient alkaline hydrogen evolution reaction. Nanoscale 2015, 7, 3130-3136.

(4) He, C.; Bo, T.; Wang, B.; Tao, J. RGO induced one-dimensional bimetallic carbide nanorods: An efficient and $\mathrm{pH}$-universal hydrogen evolution reaction electrocatalyst. Nano Energy 2019, 62, 85-93.

(5) Jin, H.; Chen, J.; Mao, S.; Wang, Y. Transition metal induced the contraction of tungsten carbide lattice as superior hydrogen evolution reaction catalyst. ACS applied materials \& interfaces 2018, 10, 22094-22101

(6) He, C.; Tao, J. Two-dimensional $\mathrm{Co}_{3} \mathrm{~W}_{3} \mathrm{C}$ nanosheets on graphene nanocomposition: An Pt-like electrocatalyst toward hydrogen evolution reaction in wide $\mathrm{pH}$ range. Mater. Today Energy 2018, 8, 65-72.

(7) Hu, Y.; Yu, B.; Ramadoss, M.; Li, W.; Yang, D.; Wang, B.; Chen, Y. Scalable synthesis of heterogeneous $\mathrm{W}-\mathrm{W}_{2} \mathrm{C}$ nanoparticle-embedded CNT networks for boosted hydrogen evolution reaction in both acidic and alkaline media. ACS Sustain. 
Chem. Eng. 2019, 7, 10016-10024.

(8) Ma, Y.-Y.; Lang, Z.-L.; Yan, L.-K.; Wang, Y.-H.; Tan, H.-Q.; Feng, K.; Xia, Y.-J.; Zhong, J.; Liu, Y.; Kang, Z.-H.; Li, Y.-G. Highly efficient hydrogen evolution triggered by a multi-interfacial Ni/WC hybrid electrocatalyst. Energy Environ. Sci. 2018, 11, 2114-2123.

(9) Lin, H.; Liu, N.; Shi, Z.; Guo, Y.; Tang, Y.; Gao, Q. Cobalt-doping in molybdenum-carbide nanowires toward efficient electrocatalytic hydrogen evolution. Adv. Funct. Mater. 2016, 26, 5590-5598.

(10) Liu, Z.; Zhang, X.; Song, H.; Yang, Y.; Zheng, Y.; Gao, B.; Fu, J.; Chu, P. K.; Huo, K. Electronic modulation between tungsten nitride and cobalt dopants for enhanced hydrogen evolution reaction at a wide range of $\mathrm{pH}$. ChemCatChem 2020, 12, 2962-2966.

(11) $\mathrm{Wu}, \mathrm{C}$; $\mathrm{Li}$, J. Unique hierarchical $\mathrm{Mo}_{2} \mathrm{C} / \mathrm{C}$ nanosheet hybrids as active electrocatalyst for hydrogen evolution reaction. ACS Appl. Mater. Interfaces 2017, 9 (47), 41314-41322

(12) Kou, Z.; Wang, T.; Wu, H.; Zheng, L.; Mu, S.; Pan, Z.; Lyu, Z.; Zang, W.; Pennycook, S. J.; Wang, J. Twinned tungsten carbonitride nanocrystals boost hydrogen evolution activity and stability. Small 2019, 15, 1900248.

(13) Chen, Z.; Wu, R.; Liu, Y.; Ha, Y.; Guo, Y.; Sun, D.; Liu, M.; Fang, F. Ultrafine Co nanoparticles encapsulated in carbon-nanotubes-grafted graphene sheets as advanced electrocatalysts for the hydrogen evolution reaction. Adv. Mater. 2018, 30, 1802011. 
(14) Jin, H.; Zhang, H.; Chen, J.; Mao, S.; Jiang, Z.; Wang, Y. A general synthetic approach for hexagonal phase tungsten nitride composites and their application in the hydrogen evolution reaction. J. Mater. Chem. A 2018, 6, 10967-10975. 